\title{
Why and How Is Compassion Necessary to Provide Good Healthcare? Comments From an Academic Physician
}

\author{
Comment on "Why and How Is Compassion Necessary to Provide Good Quality Healthcare?"
}

\author{
Christos Lionis*
}

\section{Abstract}

This is a short commentary to the editorial issued by Marianna Fotaki, entitled: "Why and how is compassion necessary to provide good quality healthcare." It introduces the necessity of a more cognitive approach to explore further the determinants of behavior towards compassionate care. It raises questions about the importance of training towards a more patient-care and values driven healthcare system.

Keywords: Compassionate Care, Undergraduate Training, Theory of Planned Behavior

Copyright: @ 2015 by Kerman University of Medical Sciences

Citation: Lionis C. Why and how is compassion necessary to provide good healthcare? Comments from an academic physician: Comment on "Why and how is compassion necessary to provide good quality healthcare?" Int J Health Policy Manag. 2015;4(11):771-772. doi:10.15171/ijhpm.2015.132

\author{
Article History: \\ Received: 5 June 2015 \\ Accepted: 13 July 2015 \\ ePublished: 15 July 2015 \\ View Video Summary

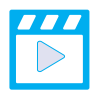 \\ *Correspondence to: \\ Christos Lionis \\ Email: lionis@galinos.med.uoc.gr
}

$\mathrm{I}$ read with interest the editorial on "Why and how is compassion necessary to provide good healthcare?" by Marianna Fotaki. ${ }^{1}$ To approach those fundamental questions, the author accepts that compassion is a foundation of ethics which guide healthcare professionals and a basis of ethics of care in health services organizations. She attempted to support this assumption by utilizing aspects from moral philosophers and political theorists. By this linkage between compassion and ethics the author suggested that caring with compassion could be inculcated through health education by considering the interrelation between individual motivation and social structure. On this basis, a cognitive approach may be utilized to explore this interrelation as an important determinant of the healthcare practitioner's intention to behave (care) with compassion. The theory of planned behavior ${ }^{2}$ seems to offer a suitable ground for such an approach. According to this theory: "human behavior is guided by three kinds of considerations: beliefs about the likely outcomes of the behavior and the evaluations of these outcomes (behavioral beliefs), beliefs about the normative expectations of others and motivation to comply with these expectations (normative beliefs), and beliefs about the presence of factors that may facilitate or impede performance of the behavior and the perceived power of these factors (control beliefs)." It would be worthwhile to explore further as to how we can modify the perceived behavior of healthcare practitioners with a focus on general practitioners/family physicians. To what extent we can enhance the intention to behave by providing teaching courses or by the establishment of incentives remains to be discussed.

Likewise, do we have evidence that we can modify the professional behavior of healthcare practitioners by changing their belief system towards the moral dimension of medical practice, by intervening in the current social norms of the medical profession, or by convincing them that a new legitimate or incentives-based setting will enhance their probability in compassionate performing? I am afraid that the establishment of the implementation of compassionate care today seems utopian, especially in the case of healthcare practitioners who are working in settings where a 'compassionate culture' is still lacking. A culture change that places the patient at the heart of all decision-making procedures relevant to his/her health seems to be a key issue. This can perhaps be achieved by involving patients in issues relevant to the quality of care services and safety, both basic components of a patient centered approach that has been declared in certain settings in the United States ${ }^{3}$ and Europe. Likewise, a compassionate culture should be extended to include a compassionate approach towards healthcare professionals themselves. However, such an approach assigns many responsibilities to both health policy-makers and leaders or managers within the healthcare setting to change the culture within their organizations. In recent years, much discussion has been focused on the effectiveness of change in organizational culture alongside structural reforms on the improvement of performance in healthcare. However, a systematic review by Parmelli et $\mathrm{al}^{4}$ failed to identify any effective and generally targeted strategies to change organizational culture. Thus, further discussion is required alongside research at a national setting level in order to offer effective ideas and proposals.

Fotaki also attempts to identify the primary focus of compassion through reference to psychological literature. She underlined that: "for healthcare professionals compassion is more about relational and interpersonal practice." I agree with this statement but I still wonder if one can clearly discover such a message within the medical undergraduate training internationally. How many medical schools across 
the world currently include compassion as an independent course? Within the Medical Faculty at the University of Crete, Heraklion, Greece an attempt to teach medical students compassionate care has succeeded to attract the interest of many medical students, ${ }^{5}$ therefore, the introduction of this subject is anticipated to contribute to undergraduate medical education and turn it into a relational and interpersonal practice.

By reading this editorial and its conclusions, I have seen that the author considers that the provision of adequate training of compassion to healthcare professionals would be effective only if it is followed by specific measure and policies. Subsequently, she suggests some measures that address both the healthcare providers as individuals and the policy-makers as mediators of the healthcare system. Nevertheless, is not clear to me what is meant by 'adequate training' or "specific measures and policies." To what extent does this statement refer to undergraduate medical education as a key tool to teach empathy and compassion, or does it cover only vocational training aimed at mobilizing the hidden or invisible humanity? The question "to what extent compassion can be taught and how it can be taught" deserves attention and evidence. Blane and Mercer ${ }^{6}$ argue that: ' $a$ focus on empathy rather than compassion is likely to be most useful both theoretically and practically. Although literature in this area is still limited, there are positive signals arising from the academic setting, where initiatives are being taken for the introduction and implementation of compassionate care courses such as that on Crete, ${ }^{5}$ although this course addresses only medical students and it does not satisfy the issue of interdisciplinarity. I agree with the author that specific measures and policies are needed, and I endorse this statement. I fully agree that the financial incentives to foster the performance of compassion are not the solution, although practicing with compassion may lead to better clinical outcomes and consequently compassionate care may indirectly be related with such incentives in systems where quality standards and pay for performance have been established.

I share the author's opinion that despite the individual related factors involved in promoting compassionate care, we need to look beyond individuals. This statement is in agreement with a statement by Cole-King and Gilbert.7 They support that "to grow compassionate patient-centered healthcare we will also have to look beyond the individual." They further mention that social groups and cultures can greatly influence practice and values. Likewise, Robin Youngson also draws attention to a culturally and socially based approach for enabling compassionate care. ${ }^{8}$

It is true that the question posed by this editorial is difficult to be answered and it deserves more discussion at an interdisciplinary level. Medicine, as an excellent art, needs an additional approach. We need to review its content, methods and practicalities in order to teach medical students. We are entering into a period where therapists seem to be needed more than medical practitioners. Compassionate care could also be penetrated with spiritual care and motivational interviews towards a more patient-centered approach and this is another issue worthy of attention. "The global strategy on people-centered and integrated health services that builds on the lessons learnt from recent decades offers an excellent chance to put forward for comprehensive health system design" as Dr. Edward Kelley says in the forward of a World Health Organization (WHO) Interim Report, ${ }^{9}$ I agree but towards a more compassionate healthcare system.

In conclusion, this editorial clearly expresses both in its title and in its epilogue a key statement which induces an important impact on clinical practice in many settings, not only within the National Health Service (NHS) in the United Kingdom. The current economic crisis has affected many European countries and has caused a strong negative impact on both healthcare services and health professionals. This current situation presents a reason to proceed with urgent action towards a new way of thinking regarding provision of services. Compassionate care may be the trigger that could guide affected healthcare systems towards a new orientation, while professional values and healthcare services should be reconsidered in terms of the development of a more patient centered care approach.

\section{Ethical issues}

Not applicable.

Competing interests

Author declares that he has no competing interests.

Author's contribution

$C L$ is the single author of the manuscript.

\section{References}

1. Fotaki M. Why and how is compassion necessary to provide good quality healthcare. Int J Health Policy Manag. 2015;4(4):199204. doi:10.15171/ijhpm.2015.66

2. Ajzen I. Behavioral Interventions Based on the Theory of Planned Behavior: Brief Description of the Theory of Planned Behavior. http://people.umass.edu/aizen/pdf/tpb.intervention. pdf. Published 2006.

3. American Academy of Family Physicians (AAFP), American Academy of Pediatrics (AAP), American College of Physicians (ACP), American Osteopahic Association (AOA). Joint principles of the patient-centered medical home. http://www.aafp.org/ dam/AAFP/documents/practice_management/pcmh/initiatives/ PCMHJoint.pdf. Accessed December 1, 2014. Published 2007.

4. Parmelli E, Flodgren G, Beyer F, Baillie N, Schaafsma ME, Eccles MP. The effectiveness of strategies to change organizational culture to improve healthcare performance: a systematic review. Implement Sci. 2011;6:33. doi:10.1186/1748-5908-6-33

5. Lionis $C$, Shea S, Markaki A. Introducing and implementing a compassionate care effective for medical students in Crete. Journal of Holistic Healthcare. 2011;8:38-41.

6. Blane D, Mercer S. Compassionate Healthcare: is empathy the key? Journal of Holistic Healthcare. 2011;8:18-21.

7. Cole-King A, Gilbert P. Compassionate care: the theory and the reality. Journal of Holistic Healthcare. 2011;8:29-35.

8. Youngson R. Talking off the armor. IIIn Crises Loss. 2010;18:7982.

9. World Health Organization (WHO). WHO global strategy on people- centered and integrated health services: Interim Report. Geneva: WHO; 2015 